\title{
EL SECTOR ELÉCTRICO EN CHILE: DESAFÍO PARA LA INNOVACIÓN Y DESARROLLO
}

\author{
Rodrigo Palma Behnke
}




\section{RODRIGO PALMA BEHNKE}

Profesor asociado, académico jornada completa del Departamento de Ingeniería de la Facultad de Ciencias Físicas y Matemáticas de la Universidad de Chile. Ingeniero Civil de Industrias con mención en Electricidad, magíster en Ciencias de la Ingeniería de la Pontificia Universidad Católica de Chile y doctor en Ingeniería de la Universidad de Dortmund, Alemania. Su campo de investigación se centra en la planificación y operación de sistemas eléctricos de potencia en mercados competitivos, las energías renovables y el desarrollo de herramientas de apoyo a la docencia. Dicta las cátedras de Sistemas de Energía, Mercados Energéticos y Taller de Proyecto en Energías Renovables. Es coautor del libro Los sistemas eléctricos de potencia (Nom Lüfke), publicado por Prentice Hall. Es senior member del IEEE y presidente de la sección Chile del IEEE (períodos 2003-2004, 2011-2012). Ha sido miembro del Comité de Expertos y Panel de Expertos de la Ley General de Servicios Eléctricos en Chile. Participó en la Comisión Asesora de Desarrollo Eléctrico (CADE), constituida por el gobierno de Chile. Es director del Centro de Energía de la Facultad de Ciencias Físicas y Matemáticas de la Universidad de Chile y director del Centro de Excelencia FONDAP en Energía Solar, SERC-Chile. 


\section{EL SECTOR ELÉCTRICO EN CHILE: DESAFÍO PARA LA INNOVACIÓN Y DESARROLLO}

\section{INTRODUCCIÓN}

El país ha enfrentado permanentemente desafíos en el sector eléctrico. En un inicio, se trató de la necesidad de electrificar el país, tema que fue abordado con la creación de la ENDESA en la década del 40. De esta manera, sistemas eléctricos aislados fueron constituyendo lo que hoy conocemos como el Sistema Interconectado del Norte Grande (SING) y el Central (SIC). Un segundo cambio estructural del sector se experimenta en la década del 80 , donde se introduce competencia en el sector eléctrico. Se lleva a cabo una desintegración vertical del sector, separando las actividades de generación, transmisión y distribución de energía eléctrica. Se formula el desarrollo de un mercado competitivo de generación, donde diversas empresas compiten en el suministro de electricidad a clientes libres y regulados. El Estado mantiene un rol subsidiario, fijando las políticas y marco normativo del sector. En lo fundamental, esta estructura de dos sistemas interconectados (SIC, SING y sistemas aislados en la zona sur) y un mercado eléctrico competitivo en generación se mantienen hasta el día de hoy.

Adicionalmente, desde el punto de vista del suministro de energía y del desarrollo país, estamos enfrentando desafíos en los siguientes ámbitos: lograr desacoplar nuestro crecimiento económico del consumo de energía; oposición y consiguiente judicialización de proyectos de generación; logros efectivos de políticas de eficiencia energética; introducción a la matriz de energías renovables disponibles en nuestro territorio; asegurar la existencia de competencia en el sector; necesidad de perfeccionar el marco normativo del sector, entre otros aspectos [1]. Estos desafíos han sido detectados y analizados a través de distintas comisiones, con apoyo de actores del sector académico, de la sociedad civil y del sector privado [2, 3].

La universidad no se considera ajena a estos fenómenos, impulsando a partir de sus académicos, distintas iniciativas tendientes a entregar elementos de juicio y soluciones en el ámbito de la energía. En este artículo se busca identificar algunas de las oportunidades de innovación y desarrollo para Chile en el sector eléctrico, y destacar la contribución que puede realizarse desde la universidad. No se pretende ser exhaustivo en esta revisión, sino más bien ejemplificar elementos de análisis transversales.

\section{ANTECEDENTES PREVIOS AL ANÁLISIS}

Hace diez años, en diciembre de 2003, junto a ocho académicos de la universidad, en un artículo para el Anuario de la Universidad de Chile, concluíamos lo siguiente en relación a la política energética y desarrollo del sector eléctrico chileno [4]: 
"En sintesis, una política energética que apunte a un sistema eléctrico sustentable debe adoptar la eficiencia energética como una de sus opciones estratégicas, para lo cual se deberán introducir mecanismos destinados a superar las barreras que impiden un pleno funcionamiento del mercado en este campo, ello supone recurrir a incentivos que reduzcan el sesgo del costo inicial, normas técnicas y ordenanzas municipales, apoyo a la IED en este campo, introducción de incentivos a los usuarios que reduzcan la demanda máxima y/o el consumo de energía, eliminación de los incentivos a aumentar las ventas de energía, generar fondos concursables para fomentar proyectos energéticamente eficientes, promover el conocimiento de las tecnologías.

A la luz del análisis anterior se detectan las siguientes líneas de trabajo de interés en este ámbito:

- Diversificar fuentes: aumentar la cantidad de fuentes de energía primaria disponibles para el sector energético, de manera de asegurar un abastecimiento confiable y menos sujeto a los vaivenes internacionales.

- Promover energías renovables: se hace necesario tener una política activa en el uso de energías renovables. Por un lado, esto asegura la sustentabilidad del sistema en el largo plazo y, por otra parte, ayuda en forma efectiva a disminuir las emisiones de carbono a la atmósfera.

- Política activa de ahorro energético: el uso eficiente de energía es la forma más económica de ampliar la disponibilidad del recurso. Las técnicas van desde el desfase temporal de consumos hasta cogeneración y otros métodos más sofisticados.

- Marco regulatorio: Se debe tender a una regulación justa que no discrimine ningún tipo de tecnología, independiente del tamaño o la fuente primaria de energía."

Si bien en estos diez años se han observado cambios importantes en distintos ámbitos del sector eléctrico nacional, llama la atención la vigencia de varios de los temas planteados en aquella ocasión, como es el caso de la necesidad de una mayor eficiencia energética y la promoción de las energías renovables.

En el año 2011, por encargo del Presidente de la Republica, la Comisión Asesora para el Desarrollo Energético (CADE) analizó y entregó su visión a los desafíos del sector energético [1, 2]. La CADE realizó un minucioso análisis de (a) la política e institucionalidad energética-eléctrica, (b) los escenarios de la matriz energética futura, (c) los niveles de competencia del mercado eléctrico chileno y (d) los principales desafíos a nivel medioambiental y de sociedad del sector, generando un set de propuestas.

Paralelamente, en mayo de 2011, se constituyó la Comisión Ciudadana TécnicoParlamentaria para la Política y Matriz Eléctrica (CCTP), la cual entregó su visión sobre las necesidades de reformas del sector eléctrico chileno a través del documento Chile necesita una gran reforma energética $[1,3]$. Este documento tiene un alcance temático mayor al de la CADE, al abordar el sector energía como un todo y plantear reformas al modelo de desarrollo del país. 
Ambos documentos muestran coincidencias y discrepancias que han servido de base para el diálogo nacional en la materia ${ }^{1}$.

En febrero de 2012, el gobierno de Chile, a través del Ministerio de Energía, publicó la Estrategia nacional de energía (ENE) 2012-2030, la cual debería ser considerada como "una carta de navegación que entrega las directrices de la política eléctrica que seguirá el país durante los próximos años" [5].

En el marco de los desafíos del sector eléctrico, el gobierno está llevando a cabo reformas, proyectos y programas que pretenden dar solución a los desafíos que se han evidenciado a lo largo de los últimos años y que han dejado al descubierto una serie de debilidades del sector en cuanto a reforzar la seguridad, robustez y eficiencia.

Por su parte, desde la academia, se han planteado distintos ámbitos de acción que buscan promover la discusión y acciones en torno al desarrollo energético.

\section{Oferta energética}

Las centrales que se encuentran operativas en el SIC suman, a diciembre de 2012, una potencia instalada de 13.545 MW (potencia bruta, no se descuentan consumos propios de las centrales). El SIC cuenta con tres cuartos de la potencia instalada a nivel nacional y abastece a más del 90\% de la población [1,7]. Por su parte, las centrales que se encuentran operativas en el SING suman una potencia instalada de 4.581 MW (potencia bruta a diciembre 2012, no se descuentan consumos propios de las centrales) [1, 7]. El SING cuenta con un cuarto de la potencia instalada a nivel nacional y, a diferencia del resto país, está constituida casi exclusivamente por centrales termoeléctricas. Los sistemas de Aysén y Magallanes poseen potencias instaladas menores con 50 MW y 101 MW, respectivamente. Lo anterior entrega una capacidad instalada total de 18.277 MW, lo que, descontados los consumos propios, constituye una capacidad instalada neta en torno a los 17.000 MW. Suponiendo un crecimiento de la demanda del orden del 4\% anual, el sistema requiere adicionar unos $400 \mathrm{MW}$ de capacidad instalada todos los años en tanto no logremos desacoplar el crecimiento del consumo energético. Este es, sin duda, uno de los principales desafíos del sector.

En los dos sistemas (SIC, SING) no se han observado cambios de gran importancia en términos estructurales, más allá de la entrada en operación, desde el año 2012, de centrales carboneras que han tenido un impacto importante en la evolución reciente de los costos marginales: Ventanas IV (150 MW) de AES Gener, Campiche (270 MW) de AES Gener, Santa María de Colbún (342 MW), Bocamina II de ENDESA (350 MW).

Grandes centrales hidroeléctricas, con estudios de factibilidad avanzados, como Neltume (490 MW), Choshuenco (125 MW), Puelo (210 MW), Alto Maipo (531 MW), etc., aún no concretan su etapa de construcción. Estos proyectos se encuentran en

1. La plataforma de Escenarios Energéticos elaboró un análisis comparativo de ambos documentos disponible en http://escenariosenergeticos.cl/wp-content/uploads/2012/01/RCMAG3.pdf. 
distintas etapas de avance, observándose en todos ellos oposición por parte de la comunidad. Lo anterior no permite asegurar su ejecución.

Por su parte, las sentencias judiciales que llevaron a suspender proyectos termoeléctricos como la central Castilla (2.000 MW) ha significado una luz de alerta a otros proyectos de generación, creando incertidumbre de abastecimiento y de costos operacionales a proyectos mineros en carpeta.

La matriz de Energías Renovables No Convencionales (ERNC) alcanza un valor de 1.056 MW, incluyendo 4,8 MW en iniciativas solares y eólicas aisladas (ver cuadros siguientes, [8]).

Figura 1

ESTADÍSTICAS ERNC

(arriba: estado de proyectos; abajo: \% capacidad instalada a nivel país)
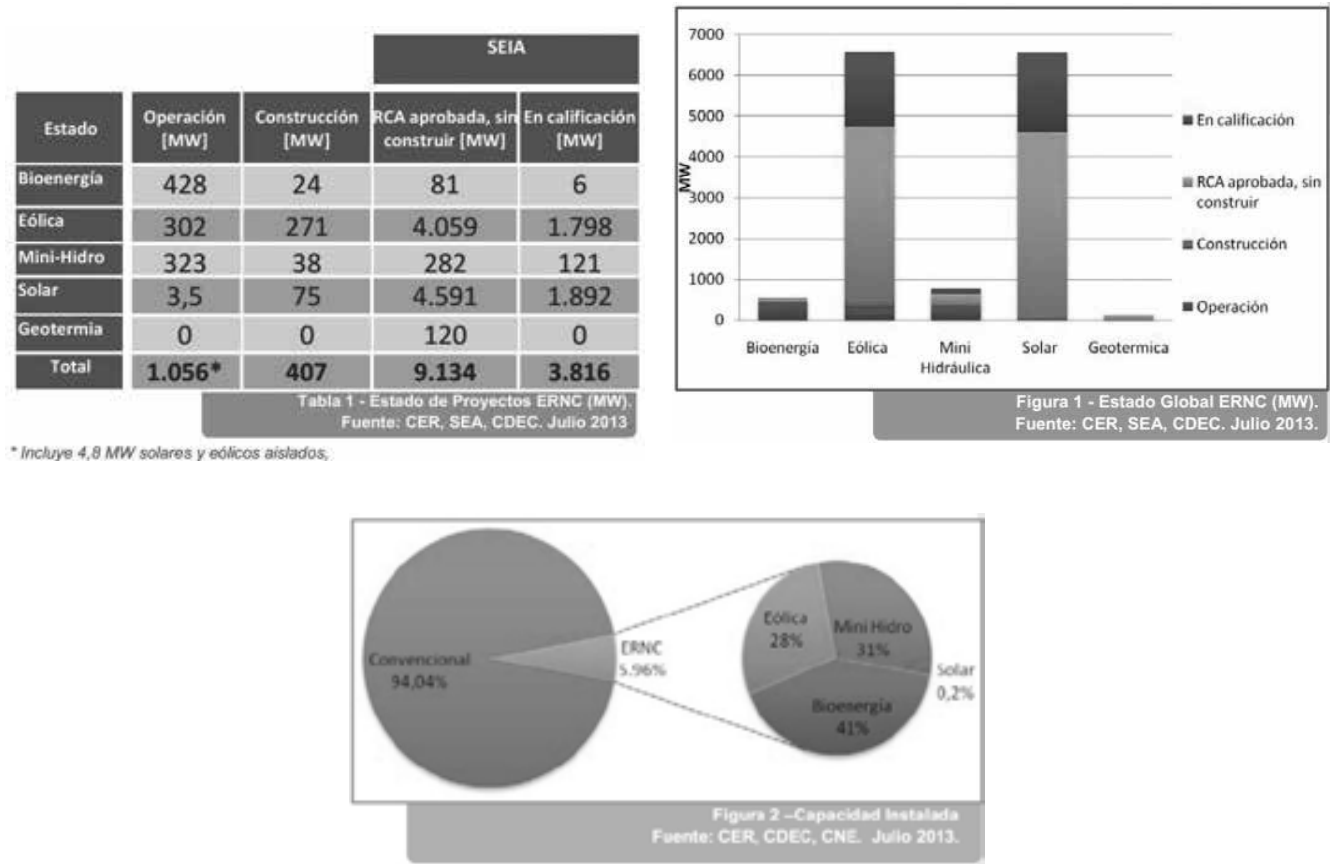

El alto interés en el desarrollo de proyectos ERNC, con 9.134 MW aprobados en el Sistema de Evaluación de Impacto Ambiental (SEIA), contrasta con un porcentaje bajo de proyectos en construcción ( $407 \mathrm{MW}$ ), aspecto que debe ser considerado al momento de desarrollar proyecciones. Este contraste tiene orígenes diversos: alto porcentaje de anuncios concentrados en los últimos dos años, por lo que los proyectos aún se encuentran en fase de desarrollo; especulación por parte de actores de mercado para posicionamiento en el tema o rentar el derecho del proyecto; disponer de proyectos a la espera de ocasiones propicias de oportunidades de contratos de largo plazo a precios atractivos; espera de acciones del gobierno en relación a nuevas metas de penetración y licitaciones 
específicas, entre otras. En el primer trimestre de 2013, la generación renovable no convencional alcanzó un 5,25\% de la generación total de los sistemas mayores. Estas cifras muestran que la realidad de las ERNC ha superado los límites inferiores establecidos por la Ley de Renovables (hoy en torno a un 3,5\%).

\section{Energización rural}

Los temas de energización rural en Chile son coordinados por la División Acceso y Equidad Energética del Ministerio de Energía, cuya misión es contribuir a generar condiciones que permitan a los habitantes del país un acceso equitativo a la energía, a través de la coordinación, formulación e implementación de programas, proyectos, normativas y asesoría técnica.

Para el año 2016, se espera una cobertura del 100\% en escuelas y postas rurales con energía eléctrica con soluciones de ERNC, lo que se suma a una cobertura rural actual superior al 96\%. Sin embargo, la hibridación de cerca de ochenta sistemas diesel de alto costo de operación e impacto ambiental prevalece como un desafío para el sector. En este tema se han sumado universidades (Universidad de Chile ${ }^{2}$, Universidad de Magallanes), ONG (Desafío Levantemos Chile) y empresas (Minera Collahuasi, GDF Suez, entre otras).

\section{Precios y costos}

En el siguiente gráfico se resume la evolución de los costos de la energía eléctrica en el sector eléctrico [1, 9].

\section{Figura 2}

EVOLUCIÓN DE COSTOS MARGINALES Y PRECIOS MEDIOS

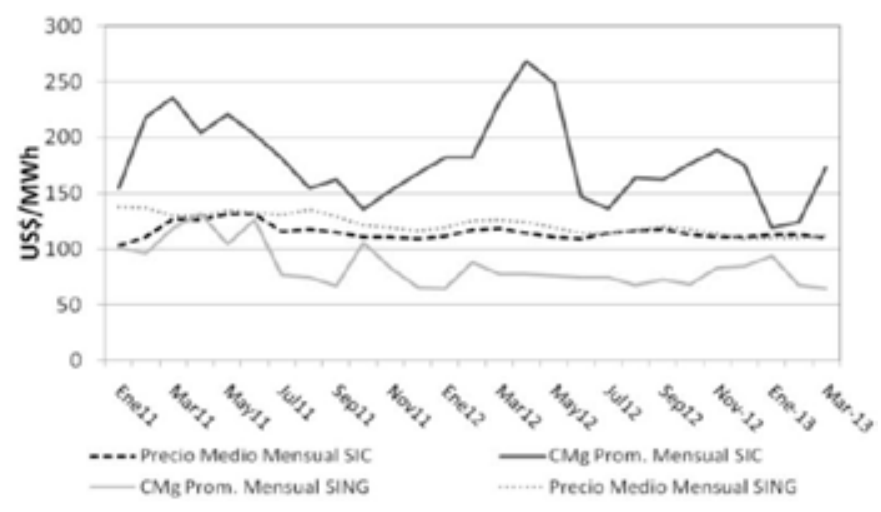

2. Proyecto Huatacondo. Véase http://www.13.cl/c/programa/factor-de-cambio/capitulos/huatacondo 
Mientras que en el SING se aprecia una tendencia sostenida a la baja en el costo marginal del sistema, lo que se justifica por la entrada en operación de las centrales carboneras descritas en la sección anterior, para el SIC, se han mantenido precios altos en torno a los 170 US\$/MWh. Esta situación de precios ha llevado a un clima de preocupación e incertidumbre en el sector que proyecta altos precios de energía que están siendo traspasados a los clientes finales y procesos productivos.

\section{OPORTUNIDADES DE INNOVACIÓN Y DESARROLLO}

En su reciente documento sobre Orientaciones estratégicas para la innovación: Surfeando hacia el futuro, Chile en el horizonte 2025, el Consejo Nacional de Innovación para la Competitividad aborda la compleja temática de la innovación para el país [6]. Se recorren las distintas aristas de la necesidad de innovar como herramienta fundamental del desarrollo y se concluye que, en este ámbito, el desafío para Chile es de carácter cultural. Para ello, se requeriría de una política de relaciones globales e inversiones -no solo del Estado, sino también académicas, empresariales e individuales- que nos permitan enriquecer nuestro capital social, pasando de la lógica de "conectarnos con el mundo" a la de "formar parte del mundo". El sector eléctrico no se encuentra ajeno al fenómeno de la innovación, distinguiéndose como uno de los elementos claves para lograr el desarrollo de nuestro país.

Cada país ha abordado de forma diferente el desafío energético. A modo de ejemplo, mediante iniciativas estratégicas se busca fortalecer una industria local que redunde en el desarrollo del país, a la vez que este esfuerzo se transforme en un nicho de exportación exitoso. Tal es el caso de Alemania, cuya opción por las energías renovables permitió la masificación de una industria eólica que trajo aparejada la creación de 250.000 puestos de trabajo. La raíz de este resultado radica en una política de incentivos por parte del gobierno que, en el caso alemán, fue un feed-in tarif (pago de la energía eléctrica inyectada al sistema a un precio predefinido) diferenciado por tecnología, es decir, se estableció un precio fijo mayor que el precio de mercado de la electricidad por la inyección de energía renovable en el sistema. Este esquema también tiene detractores, quienes a la luz de la crisis financiera que afecta a Europa cuestionan la efectividad de estas medidas. Este es el caso de España, donde empresas del ámbito de las energías renovables han quebrado o han cerrado sus unidades de producción. A esto se suma el desarrollo exponencial, en los últimos años, de la producción de tecnologías renovables en China, el que junto con impulsar a la baja los precios de estas tecnologías ha causado la pérdida de competitividad de empresas ubicadas en Europa y otros continentes.

Si bien las experiencias internacionales de países desarrollados en esta materia difícilmente pueden replicarse en la realidad de nuestro país, cabe preguntarse si existen nichos específicos en los cuales Chile pudiera realizar una apuesta de desarrollo e innovación. De ser así, tendríamos en nuestras manos la capacidad de proyectarnos como un desarrollador tecnológico a nivel regional, supliendo al mismo tiempo nuestra demanda energética interna. Con ello se le daría un sentido estratégico a la inversión de recursos, con mayor rentabilidad social y privada. 
Una opción de este tipo contrasta con la promoción de un sistema feed-in tarif, el cual podría convertirse a la larga en mero financiamiento para la adquisición de tecnología extranjera aún en fase de desarrollo y, por ende, costosa. Más aún, se podría dar el absurdo de que Chile, con un ingreso per cápita de US\$15.000, termine financiando el desarrollo de tecnologías renovables no maduras importadas desde países con ingresos por sobre los US\$ 35.000 per cápita.

Cabe preguntarse, entonces, si existen ámbitos específicos de I\&D en el sector energético del país (electrónica de potencia, microhidráulicas, geotermia, redes inteligentes, etc.), que pudieran ser impulsados como nichos de desarrollo. Para lo anterior se requiere que los actores involucrados conozcan las ventajas competitivas del país y que los investigadores y desarrolladores participantes se sientan capaces de enfrentar un desafío de esta magnitud. Efectivamente, esta apuesta de I\&D es más riesgosa que la realizada por países del primer mundo con tradición de desarrollo tecnológico. Sin embargo, ¿estamos para siempre condenados a mantenernos como país en un estado de mero comprador de tecnologías en el ámbito energético, o ya tenemos la suficiente madurez para ser desarrolladores de nuestras soluciones propias que, además, tengan perspectivas de exportación en un mundo global?

En las próximas secciones se revisan algunas de estas oportunidades, resaltando el rol que puede cumplir la academia.

\section{Proyectos I\&D, Centros de Investigación y Formación de Capital Humano}

Históricamente, el sistema de innovación nacional en el pasado no apoyó en forma sistemática y relevante la innovación tecnológica en el sector energético. En general, los proyectos apoyados por los programas FONDECYT, FONDEF o INNOVA CORFO, reciben apoyos entre $\$ 5$ millones y $\$ 100$ millones al año. El apoyo de CORFO a los programas de Biomasa con cerca de US\$ 1 millón al año, en un horizonte de cuatro años, ha marcado el inicio de apuestas de mayor alcance en este ámbito.

Sin embargo, en los últimos años se ha detectado una serie de iniciativas tendientes a abordar barreras de entrada de tecnologías ERNC y a desarrollar oportunidades de nuevos desarrollos en el ámbito de las soluciones energéticas en general. En este contexto, se puede mencionar:

- Creación reciente de Centros de Excelencia FONDAP en Energía Solar de CONICYT, denominado SERC-Chile, donde participan seis universidades y Fundación Chile, junto con el Centro de Ciencia del Clima y la Resiliencia. Estos centros poseen un financiamiento basal de US\$2 millones y tienen la misión de crear nuevos conocimientos tendientes a potenciar el desarrollo de las áreas respectivas, con un impacto en estas áreas definidas como prioritarias para el país. Asimismo, ya existe un Centro FONDAP en Geotermia (CEGA) y un nuevo llamado a crear centros de excelencia en torno al recurso agua, lo que puede tener un impacto en la forma en que podemos integrar adecuadamente nuestro recurso hidráulico a la matriz energética. 
- El programa de atracción de Centros de Excelencia CORFO ha tenido impacto en el sector energético, con la llegada de CSIRO de Australia e INRIA de Francia. En el nuevo llamado es factible que lleguen a Chile instituciones como Fraunhofer-Solar de Alemania.

- Concurso CSP (CORFO, Ministerio de Energía), que entrega subsidios directos e indirectos para la instalación de la primera planta de concentración solar de potencia en el país. Se espera una potencia en torno a los 45 MW y un importante impacto en el conocimiento de esta tecnología y la formación de capital humano.

- Proyecto Desarrollo de Capacidades Locales para Energía Solar desarrollado por el Ministerio de Energía en conjunto con el BID, financiado con recursos GEF. Este proyecto apoyará el pilotaje de paneles solares fotovoltaicos conectados a la red de distribución en colaboración con las empresas distribuidoras de electricidad del país.

- Formación de Centros de Energía en varias instituciones del país, lo que ha permitido ir formando una masa crítica de análisis en torno a los temas energéticos, como el Centro de Energías Renovables ${ }^{3}$ del gobierno en 2009, CE-FCFM ${ }^{4}$, Plataforma Solar de Atacama, CE UAI, UTFSM, CIL U Chile), etc.

En todas estas iniciativas se han detectado necesidades y abierto oportunidades de investigación y desarrollo para el país, las que pueden llegar a tener un impacto real en el desarrollo de nuestra matriz energética. Sin embargo, para los cambios estructurales que el país requiere en un mediano plazo, probablemente ninguna de estas iniciativas por sí sola permita generar los cambios necesarios. A modo de ejemplo, si decidiéramos convertir parte de nuestro desierto de Atacama en el nodo exportador de energía eléctrica para Sudamérica, no solo se requeriría del desarrollo de un conjunto idóneo de tecnologías, sería necesario establecer un nuevo esquema de desarrollo de la región, de su red de servicios, formación de capital humano especializado, una planificación territorial, entre otros temas de relevancia.

La formación de capital humano calificado nos convoca directamente como universidad, donde cabe destacar los siguientes ámbitos:

- Becas Chile ha promovido estudios y programas de magíster y doctorado en temas de energía: ERNC, Energía Nuclear, Redes Inteligentes, etc. Este esfuerzo debe continuar, en particular, en lo referido a la reinserción de estos especialistas. Se requiere del desarrollo de programas creativos de inserción en la academia, empresas productivas y sector público de este conjunto de profesionales.

- Las pasantías en el extranjero de alumnos y profesionales potencian ideas y proyectos exitosos en el ámbito de la energía que el país debe seguir proporcionando.

3. http://www.cer.gob.cl

4. http://www.centroenergia.cl 
- Asimismo, se ve como positivo el fomento a la formación de redes entre centros de investigación nacionales y con el extranjero. Las dinámicas de cooperación abren oportunidades de proyectos e iniciativas conjuntas que son difíciles de detectar anticipadamente.

- Se requiere de la creación de una generación de innovadores que puedan liderar los procesos que requiere el sector eléctrico. No es evidente la forma en que se puede contribuir a este objetivo. Sin embargo, las claves pueden encontrarse a través de la adaptación de nuestras mallas curriculares, contenidos y métodos utilizados en cátedra, acercamiento al mundo industrial, fomento de actividades interdisciplinarias y competencias en torno a desafíos tecnológicos.

\section{Apoyo a la definición de matriz energética}

Habiéndonos generado la convicción de que el tema energía ha dejado de ser un problema meramente técnico y que, en realidad, nos involucra a todos como sociedad, es de interés reconocer que a nivel nacional existen distintos esfuerzos, desarrollos en esta materia, los que se resumen a continuación:

- Iniciativa de Escenarios Energéticos en su segunda versión denominada Energía Presidencial. Esta iniciativa, que entregó los resultados de su segunda versión en julio de $2013^{5}$, corresponde a un proceso participativo de análisis iniciado en el año 2009 donde, en un marco común de modelación, se evaluaron distintas visiones de desarrollo de la matriz energética del país. En su versión 2012, se seleccionaron dos visiones de desarrollo: "Chile Sustentable" y "Universidad Adolfo Ibáñez", las que son comparadas y sensibilizadas respecto de distintos parámetros de interés definidos por las cerca de veinticinco instituciones públicas, privadas y de la sociedad civil que participan del proceso. Esta iniciativa ha logrado convocar a distintos actores del sector, normalmente ubicados en veredas opuestas, constituyéndose en un punto de encuentro para la discusión sobre los temas de desarrollo de la matriz energética de Chile. Esta situación abre una oportunidad de profundizar la discusión a partir de la plataforma de comunicación ya creada, donde la universidad puede contribuir, por ejemplo, con formas de análisis, esquemas de interacción, modelos y algoritmos de solución.

- Proyecto MAPS-Chile ${ }^{6}$ liderado por el Ministerio del Medioambiente y con la participación de otros seis ministerios, se refiere a un proyecto gubernamental a dos años plazo (2012-2013), que genera, analiza y valida información, modelación e investigación sobre las trayectorias de emisiones de gases de efecto invernadero en Chile. Asimismo, identifica diversas acciones para la mitigación de las emisiones en el país, que sean compatibles con las estrategias nacionales

5. http://www.escenariosenergeticos.cl

6. http://www.mapschile.cl 
de desarrollo. Se busca, hacia finales del año 2013, proponer posibles medidas (de política pública y privada) que permitan avanzar en la mitigación y en el compromiso internacional de reducción de emisiones del país. Entre estas medidas pueden mencionarse aquellas que impactan la matriz energética por la vía de impuestos o metas de penetración de energía renovable. En su desarrollo participan especialistas e individuos de sectores diversos con experiencia en el tema. Asimismo, se cuenta con el apoyo internacional de profesionales que han desarrollado proyectos similares (MAPS en Sudáfrica, por ejemplo). En este ámbito, es fundamental que las universidades puedan crear grupos de investigación y desarrollo capaces de desarrollar modelos y herramientas con capacidades interdisciplinarias, que sirvan de sustento para acompañar un proceso participativo con distintas visiones y formas de abordar el problema del cambio climático.

- Estudios de GIZ para el SIC-SING del Ministerio de Energía. La Agencia de Cooperación Técnica de Alemania ha desarrollado, desde el año 2002, una serie de estudios tendientes a abordar las barreras de desarrollo que enfrentan las ERNC en Chile. Se han realizado estudios de penetración de ERNC en el SIC y SING que permiten dimensionar los costos de escenarios ERNC, distinguiendo, asimismo, efectos de corto plazo y de la operación dinámica del sistema. Estos problemas no están resueltos a nivel internacional, por lo que requieren de la creación de capacidades locales en las universidades e instituciones relacionadas (Centros de Despacho Económico de Carga, Comisión Nacional de Energía, ministerios, empresas) que entreguen respuestas aplicables a nuestra realidad específica.

Asociado al punto anterior, se requiere de un apoyo al desarrollo de modelos ajustados para Chile que permitan integrar el sector energía en el estudio de los efectos de políticas públicas, medidas de mitigación, etc. Lo anterior requiere de un proceso de validación participativa entre los actores del mercado. Es deseable que estos modelos se transformen en bienes públicos, es decir, que su uso sea de acceso gratuito para los tomadores de decisión. Esta es una tendencia observada en países como Nueva Zelanda y Colombia, donde es factible usar modelos que permiten estimar los costos del sistema, peajes, riesgos, etc.

En relación a la matriz energética futura se detecta un distanciamiento progresivo de la sociedad chilena con el recurso agua, que históricamente ha jugado un rol central en el desarrollo de la oferta de energía eléctrica y requerimientos de regadío. De un pasado donde los grandes proyectos hidráulicos correspondían a iniciativas del Estado, en una época donde esto le entregaba una legitimidad y aceptación ante la sociedad, se ha migrado a un conjunto de iniciativas privadas, donde la comunidad percibe más perjuicios que beneficios. Es urgente poder encontrar fórmulas donde el recurso hidráulico del país nuevamente sea percibido como un aporte directo a su desarrollo por parte de todos los agentes: público, privados y sociedad civil. Entre los aspectos a analizar destacan el manejo, costo, duración y propiedad de los derechos de agua, todos temas que requieren de análisis profundos y propuestas creativas. 
El resultado de estas iniciativas, junto con estudios específicos, pueden ser una contribución para definir políticas públicas y marcos normativos efectivos.

\section{Desarrollos tecnológicos específicos}

La innovación tecnológica juega un rol central en los desafíos energéticos de países desarrollados. Chile ha tomado conciencia de la importancia que posee la innovación para su desarrollo futuro. El sector energía no está ajeno a este fenómeno. Si bien se reconoce que es muy complejo formar parte de un proceso de innovación de impacto mundial en el tema energético, debido al costo intensivo de su desarrollo, existe un potencial importante de desarrollo de innovación en nichos específicos [1, 6]. Algunas de estas iniciativas se pueden resumir en los siguientes puntos:

- Se deberá poner atención al desarrollo de las baterías, acumuladores o sistemas de almacenamiento de energía, que pueden solucionar una de las principales complicaciones que presentan algunas fuentes de energía limpia (como la solar y la eólica) que es la variabilidad de su producción:

- La perspectiva de escasez de combustibles fósiles y de desarrollo de los vehículos eléctricos a nivel mundial ha impulsado el interés por el mercado del litio, elemento esencial en el desarrollo de acumuladores de alta densidad energética (volumen y peso). Desde el punto de vista nacional, este tema cobra relevancia por las reservas de litio de que disponemos. El 83\% de las reservas mundiales se encuentran en Argentina, Chile y Bolivia. Actualmente, el mercado del litio mueve US $\$ 800$ millones al año a nivel mundial. Una cifra no muy relevante si se la compara con otras actividades mineras. Sin embargo, quienes abogan por la importancia estratégica de este mineral señalan que en los últimos dos años este negocio ha crecido a tasas del 12\% y en el futuro la demanda de litio, impulsada principalmente por la industria tecnológica, hará de su explotación un negocio atractivo en donde la demanda aumentaría hasta los US\$1.000 millones hacia 2020, y unos US\$ 2.000 millones al año 2030 [10]. A nivel académico, junto a otras iniciativas, la Universidad de Chile ha creado el Centro de Innovación del Litio ${ }^{7}$, el que busca incursionar en la cadena de valor del litio identificando oportunidades I\&D y emprendimiento tecnológico en el país. La discusión pública y política que se ha dado respecto al litio en su estrategia de explotación, ha permitido levantar una discusión paralela respecto al valor agregado y la necesidad de aprovechar este nuevo paradigma energético en desarrollar ciencia y tecnología. En el caso de Chile, los sistemas de acumulación a base

7. http://www.pcil.cl 
de litio se proyectan en forma natural como la tecnología que permite compensar las variaciones de algunas energías renovables con alto potencial.

- Las centrales de bombeo, ya en la perspectiva de empresarios nacionales como es el caso de la empresa Valhalla, también pueden ofrecer una alternativa eficiente para situar a Chile como un nodo de desarrollo de soluciones de energías renovables variables (eólica y solar). El bombeo de agua de mar a un embalse ubicado en altura, durante las horas de exceso de energía renovable, permite un uso económico de esta energía al poder aprovecharla a través de turbinas generadoras de electricidad en las horas en que la energía renovable no esté disponible. Este complemento a la energía variable puede sumarse a soluciones de respaldo basadas en turbinas a gas natural, en la medida en que este combustible esté disponible a costo competitivo para nuestro país.

- Se ha detectado que las redes inteligentes pueden contribuir de manera relevante a una integración costo-efectiva de la ERNC en las redes eléctricas nacionales y a optimizar el uso de la infraestructura de transmisión. Respecto de la infraestructura de redes de transmisión y equipos de generación, se busca incluir componentes de control y monitoreo remoto y automatizado (SCADA), de manera de mejorar los tiempos de respuestas y calidad de la regulación.

- Introducir soluciones de distribución a nivel rural que hagan uso de los conceptos de redes inteligentes. A este tipo de soluciones se lo denomina "microredes", lo que debiera ser la base para hibridizar sistemas rurales. La idea central es permitir una coexistencia de sistemas diesel (de fácil operación y disponibilidad programada de la fuente de energía) con energía disponible localmente (ERNC solar, biomasa, hidráulica, eólica, etc.). La microred permite asegurar una operación confiable técnicamente y de bajo costo de mantenimiento en la medida que exista un involucramiento real de la comunidad.

- Apoyo en la creación de programas específicos focalizados en estas áreas, con cierta continuidad temporal y magnitudes de recursos que permitan su desarrollo a nivel piloto o demostración. Concretamente, se distinguen oportunidades en soluciones de cogeneración solar eléctrica-térmica para la minería, geotermia en altura, biocombustibles específicos, microhidráulica, maremotriz, tratamiento solar de agua, entre otras. Se debería promover desarrollos a nivel piloto con financiamientos adecuados que permitan validar su escalamiento. A partir de ello, debería promocionarse activamente el derribar barreras que bloqueen el desarrollo de estos emprendimientos.

\section{CONCLUSIONES}

Si bien el sector eléctrico siempre ha sido gravitante en el desarrollo del país, producto del impacto que posee el uso de la energía en nuestras actividades diarias y en los procesos productivos, hoy estamos viviendo una situación especialmente incierta en relación 
a los precios de la energía y al desarrollo de nuestra matriz. El problema de la energía ha dejado de estar centrado en lo técnico, dando paso a la necesidad de una mirada interdisciplinaria como única alternativa para entender su complejidad. En este contexto, la innovación juega un rol central en los desafíos energéticos que enfrenta nuestro país. Por su parte, la universidad es convocada a hacerse parte de este proceso.

Este artículo presenta un acercamiento a esta problemática, con una breve descripción del sector eléctrico y su desarrollo histórico, y presentando los desafíos actuales junto con los ámbitos de estudio requeridos.

Por último, a través de ejemplos específicos de iniciativas, se pretende graficar las posibles acciones que pueden ser fomentadas o apoyadas desde la universidad. Cabe señalar que este análisis no pretende ni puede ser exhaustivo, por lo que se espera sea una motivación para futuras contribuciones en esta temática.

\section{REFERENCIAS BIBLIOGRÁFICAS}

Listado de la bibliografía citada en el estudio:

1. Palma, R., Análisis estratégico del sector energético en Chile, Banco Interamericano de Desarrollo (BID), 2013.

2. Agurto Colima, R., Alarcón Arias, I., Bernstein Letelier, S., Blanlot Soza, V., Borregaard de Strabucchi, N., Del Campo Fayet, S., Fischer Barkan, R., Guzmán Molinari, J.A., Jadresic Marincovic, A., Palma Behnke, R., Quiroz Castro, J., Rudnick Van de Wyngard, H., Tokman Ramos, M., Vicuña Díaz, S., Zanelli Iglesias, J., Informe de la Comisión Asesora para el Desarrollo Eléctrico (CADE), Santiago, noviembre 2011, versión electrónica en http://www. minenergia.cl/documentos/estudios.html.

3. Comisión Ciudadana-Técnica-Parlamentaria (CCTP), Chile Necesita Una Gran Reforma Energética, octubre 2011, versión electrónica en http://www.energiaciudadana.cl/docs/InformeCompleto.pdf.

4. Palma, R., Vargas, L., Moya, O., Arellano, S., Galetovic, A., Jofré, A., Román, R., Muñoz, A., Maldonado, P., "Política energética y desarrollo eléctrico", en Anuario de Chile 2003/4, diciembre 2003.

5. Ministerio de Energía, Estrategia Nacional de Energía 2012-2030, febrero 2012.

6. Consejo Nacional de Innovación para la Competitividad, Orientaciones estratégicas para la innovación: Surfeando hacia el futuro, Chile en el horizonte 2025, 2013.

7. Estadísticas disponibles en portal www.centralenergia.cl, mayo 2013.

8. Centro de Energías Renovables, Informe Mensual, julio 2013, versión electrónica en http:// www.cer.gob.cl.

9. Comisión Nacional de Energía, Informe de precios de nudo, SIC-SING, abril 2013.

10. Lagos, G., El desarrollo del litio en Chile: 1984-2012, Centro de Minería, PUC, agosto 2012. 